\title{
Functionalization of Carbon Nanotubes Using A Silane Coupling Agent
}

Peng Cheng MA ${ }^{1}$, Jang-Kyo $\mathrm{KIM}^{1^{*}}$ and Ben Zhong TANG ${ }^{2}$

\section{Addresses and affiliations for the authors:}

Peng Cheng MA

Tel.: +852 2358 8657; Fax: +852 2358 1543. E-mail: mapc@ust.hk.

Address: Department of Mechanical Engineering, the Hong Kong University of Science \& Technology, Clear Water Bay, Kowloon, Hong Kong, China

Jang-Kyo KIM (Corresponding Author)

Tel.: +852 2358 7207; Fax: +852 2358 1543. E-mail: mejkkim@ust.hk.

Address: Department of Mechanical Engineering, the Hong Kong University of Science \& Technology, Clear Water Bay, Kowloon, Hong Kong, China

\section{Ben Zhong TANG}

Tel.: +852 2358 7375; Fax: +852 2358 1594. E-mail: tangbenz@ust.hk.

Address: Department of Chemistry, the Hong Kong University of Science \& Technology, Clear Water Bay, Kowloon, Hong Kong, China 


\title{
Functionalization of Carbon Nanotubes Using A Silane Coupling Agent
}

\author{
Peng Cheng MA ${ }^{1}$, Jang-Kyo $\mathrm{KIM}^{1^{*}}$ and Ben Zhong TANG ${ }^{2}$ \\ ${ }^{1}$ Department of Mechanical Engineering and ${ }^{2}$ Department of Chemistry
}

The Hong Kong University of Science \& Technology, Clear Water Bay, Kowloon, Hong Kong, China

\begin{abstract}
A new method is developed to chemically functionalize multi-walled carbon nanotubes (MWCNTs) based on silanization reaction for use as the reinforcement for polymer matrix composites. To oxidize and create active moieties on the MWCNTs, the samples were exposed to UV light within the ozone chamber, followed by silanization using 3-glycidoxypropyltrimethoxy silane after the oxidized MWCNTs were reduced by lithium aluminum hydride. FT-IR, TEM and XPS were employed to characterize the changes in carbon nanotubes surface morphology, chemistry and physical conditions at different processing stages. The results indicate improved dispersion and attachment of silane molecules on the surface of the MWCNTs.
\end{abstract}

\section{Keywords:}

Carbon nanotubes; Surface treatment; Functional groups; Surface properties

\section{Introduction}

With unique structural and transport properties, such as excellent strength, modulus, electrical and thermal conductivities along with low density, carbon nanotubes (CNTs) have attracted much interest in the field of CNT/polymer nanocomposites [1-3]. These composites hold the promise of delivering exceptional mechanical properties and multi-functional characteristics. However, if these materials

\footnotetext{
* Corresponding author. Tel.: +852 2358 7207; Fax:+852 2358 1543. E-mail: mejkkim@ust.hk.
} 
are to be utilized as effective reinforcements in polymer composites, proper dispersion and good interfacial bonding between the CNTs and the polymer matrix have to be guaranteed [4]. Therefore, many different surface treatment and functionalization techniques [5] have been devised for improving the dispersion and interfacial adhesion of CNTs with polymer resins. Among these methods, high concentration acids treatment has shown promising results to provide functional groups (carboxyl groups) onto the CNTs surface. This method, however, not only creates defects on the CNT sidewalls, but also decreases the aspect ratio of CNTs because the strong acids cut the CNTs into short pieces during the oxidative process $[6,7]$. The defects severely degrade the mechanical properties of CNTs. Ozone treatment of the CNTs has been developed as an alternative, milder method $[8,9]$ where the surface of the nanotubes is decorated with active moieties, thus substantially broadening the chemical reactivity of the CNTs. These ameliorating effects have been further improved by incorporating with UV light [10].

Polysiloxane of various kinds has been widely used as coupling agents between glass substrates and polymeric resins [11]. The coupling process can be accomplished via the chemical reaction between the trialkoxy groups of silane molecules and the hydroxyl groups on the glass substrates, whereas other functional groups of silane molecules, which are generally ethylene, amine, epoxy, thiohydroxy, etc, can be remained. Recently, this process has been applied to functionalize the CNTs surface [12-14].

This paper is part of a larger project on the development of surface treatment techniques for carbon nanotubes as the reinforcement of polymer matrix nanocomposites. A new method is presented for the chemical functionalization of multi-walled carbon nanotubes (MWCNTs) through the combined process of $\mathrm{UV} / \mathrm{O}_{3}$ treatment and silanization reaction. The silane molecules containing epoxy ended-groups are covalently bonded on the surface of the MWCNTs, which in turn modifies the surface characteristics of the MWCNTs and improves the compatibility of MWCNTs with epoxy. 


\section{Experimental}

\subsection{Materials}

MWCNTs used in this study are basically the same as those employed in our previous work [10], which were prepared by a chemical vapor deposition method (supplied by Iljin Nanotech Ltd., Korea). The diameter and length ranged between $10-20 \mathrm{~nm}$ and $10-50 \mu \mathrm{m}$, respectively, according to the supplier's specification. 3-Glycidoxypropyltrimethoxy silane (GPTMS) with purity of $98 \%$ (supplied by Aldrich) was used as the silane functionalization agent. The following reagents and solvents were used without further purification: toluene $(99.5 \%, \mathrm{BDH})$, ethanol (99.9\%, Merck), methanol ( $>99.5 \%$, Fisher), acetone (99.5\%, General Chemical), and lithium aluminum hydride (powder, 95\%, Aldrich).

\subsection{Treatment of MWCNTs}

\subsubsection{Pretreatment of MWCNTs}

A two-step pretreatment of ball milling and ultrasonication was established after an extensive trial and error to disentangle and disperse the MWCNTs before $\mathrm{UV} / \mathrm{O}_{3}$ treatment. Typically, $250 \mathrm{mg}$ of MWCNTs was put into a stainless steel ball milling container with 450 pieces of $\mathrm{ZrO}_{2}$ milling beads ( 150 pieces of beads with diameter of 5.5, 3.0 and $2.0 \mathrm{~mm}$, respectively). The rolling speed was fixed at $250 \mathrm{rpm}$ and the milling was continued for $2 \mathrm{~h}$. Then $50 \mathrm{mg}$ MWCNTs were transferred into a beaker containing $60 \mathrm{ml}$ of toluene, and ultrasonically treated using a Digital Sonifier (Branson Ultrasonics Corporation, USA) for $1 \mathrm{~h}$. After the treatment, the solution was filtered through a nylon filter with pore size of $1.0 \mu \mathrm{m}$, which was then dried in a vacuum oven at $80^{\circ} \mathrm{C}$ for $4 \mathrm{~h}$.

\subsubsection{Oxidation of MWCNTs}

The aim of oxidation treatment was to create active sites and moieties on the surface of nanotubes. The filtered MWCNTs were dried at $80^{\circ} \mathrm{C}$ for $4 \mathrm{~h}$, which were then subjected to $\mathrm{UV} / \mathrm{O}_{3}$ treatment 
in a chamber (Jelight 144AX-220 UV/Ozone Cleaning System) for $2 \mathrm{~h}$. The cleaner contained a low-pressure mercury vapor grid lamp that could release ultraviolet radiation of $28 \mathrm{~W} / \mathrm{cm}^{2}$. The wavelength of the ultraviolet light was $253.7 \mathrm{~nm}$, and the distance between the UV lamp and the sample stage was fixed at $20 \mathrm{~mm}$.

\subsubsection{Reduction of MWCNTs}

The reduction of the oxidized MWCNTs was realized using lithium aluminum hydride $\left(\mathrm{LiAlH}_{4}\right)$. Typically, $20.0 \mathrm{mg}$ of oxidized MWCNTs was dispersed in toluene by ultrasonication (in a water bath) for $30 \mathrm{~min}$, and then $5.0 \mathrm{mg}$ of $\mathrm{LiAlH}_{4}$ was added gently. The solution was stirred for $1 \mathrm{~h}$ at room temperature, followed by adding $2.0 \mathrm{ml}$ of $2.0 \mathrm{~N}$ hydrochloric acid into the solution in order to remove the lithium and aluminum. The reduced MWCNTs were obtained by filtration of the solution and washing with toluene, absolute ethanol and acetone, and then drying in a vacuum oven at $80^{\circ} \mathrm{C}$ overnight.

\subsubsection{Silanization of MWCNTs}

$20.0 \mathrm{mg}$ of reduced MWCNTs was added into a three-neck flask with $50.0 \mathrm{ml}$ toluene and dispersed through ultrasonication (in a water bath) for $30 \mathrm{~min}$. Then $7.5 \mathrm{ml}$ of $1.0 \mathrm{wt} \%$ toluene solution of GPTMS was added and stirred for $6 \mathrm{~h}$ at $60-65^{\circ} \mathrm{C}$ for silanization. After the reaction, $30 \mathrm{ml}$ of methanol was added to dilute the unreacted GPTMS molecules. The product was obtained by filtration and washing with methanol, water and acetone sequentially. The silane treated MWCNTs were dried in a vacuum oven at $80^{\circ} \mathrm{C}$ for $12 \mathrm{~h}$.

\subsection{Characterization of MWCNT Surface}

Fourier transform infrared (FTIR) spectrometry, X-ray photoelectron spectroscopy (XPS) and field emission analytical transmission electron microscope (TEM) were used to characterize the changes in chemical structure and surface morphology of the MWCNTs after each surface 
treatment step. For the FTIR (Perkin Elmer Spectrum One, USA), the MWCNTs were pressed into a pellet with potassium bromide $(\mathrm{KBr})$ and scanned from $500 \mathrm{~cm}^{-1}$ to $4000 \mathrm{~cm}^{-1}$ at a resolution of 4 $\mathrm{cm}^{-1}$. TEM images were taken on a JEOL 2010F (JEOL Ltd., Japan) with an energy dispersion X-ray (EDX) analyzer. In the XPS analysis (Surface analysis PHI5600, Physical Electronics, Inc.), a monochromatic $\mathrm{Al} \mathrm{K} \alpha \mathrm{X}$-ray was used at $14 \mathrm{kV}$. The XPS curve fitting of $\mathrm{C} 1 \mathrm{~s}$ and $\mathrm{Si} 2 \mathrm{p}$ was accomplished by MultiPak V6.0 A (Physical Electronics, Inc.).

\section{Results and discussion}

\subsection{Surface Functionalities of MWCNTs}

Figure 1 shows the FTIR results of the MWCNTs obtained at different processing steps. For the pristine MWCNTs (Fig. 1A), the bands at 3419 and $1058 \mathrm{~cm}^{-1}$ are attributed to the presence of hydroxyl groups $(-\mathrm{OH})$ on the surface of the MWCNTs, which are believed to result from either ambient atmospheric moisture bound to the MWCNTs or oxidation during purification of the raw material [15]. Another band at $1626 \mathrm{~cm}^{-1}$ is assigned to the $\mathrm{C}=\mathrm{O}$ stretching of quinone groups on the surface of MWCNTs.

For the oxidized MWCNTs (Fig. 1B), two new bands appeared at 1715 and $1160 \mathrm{~cm}^{-1}$, which are attributed to the $\mathrm{C}=\mathrm{O}$ and $\mathrm{C}-\mathrm{O}$ stretching vibrations of the carboxylic groups (-COOH), respectively. The increase in the relative intensities of the bands at 1068 and $3420 \mathrm{~cm}^{-1}$ (I1068/I3420) suggests that there are more-OH groups on the MWCNT surface after the oxidation. All these observations confirm that the surface of the MWCNTs has been functionalized or activated by the $\mathrm{UV} / \mathrm{O}_{3}$ treatment.

The FT-IR spectrum of the reduced MWCNTs (Fig. 1C) shows the reduction of the -COOH groups to $-\mathrm{OH}$ groups, as verified by the disappearance of the band at $1715 \mathrm{~cm}^{-1}$ and the appearance of a band at $1384 \mathrm{~cm}^{-1}$, which is assignable to the bending vibration of the $-\mathrm{OH}$ group. 
In the silane-functionalized MWCNTs (Fig. 1D), the band at $3410 \mathrm{~cm}^{-1}$ became broader and weaker, and the band at $1384 \mathrm{~cm}^{-1}$ almost disappeared. On the other hand, two new bands at 2914 and $2848 \mathrm{~cm}^{-1}$ associated with the stretching of the methylene groups from the GPTMS molecules appeared. The weak signal at $793 \mathrm{~cm}^{-1}$ confirms the presence of the epoxy group on the GPTMS-modified MWCNTs. Although there is a possibility of reactions between -OH and epoxy group, this reaction can only be accomplished in the presence of catalysts, such as tertiary amine [16]. In our experiment, toluene was used as the reaction media, and the temperature was controlled at $60-65^{\circ} \mathrm{C}$. Therefore, the chance for reactions between $-\mathrm{OH}$ and epoxy was very small. This conclusion along with the FT-IR spectral data confirm that the $-\mathrm{OH}$ groups of the MWCNTs could react only with the $-\mathrm{OCH}_{3}$ groups of the GPTMS molecules.
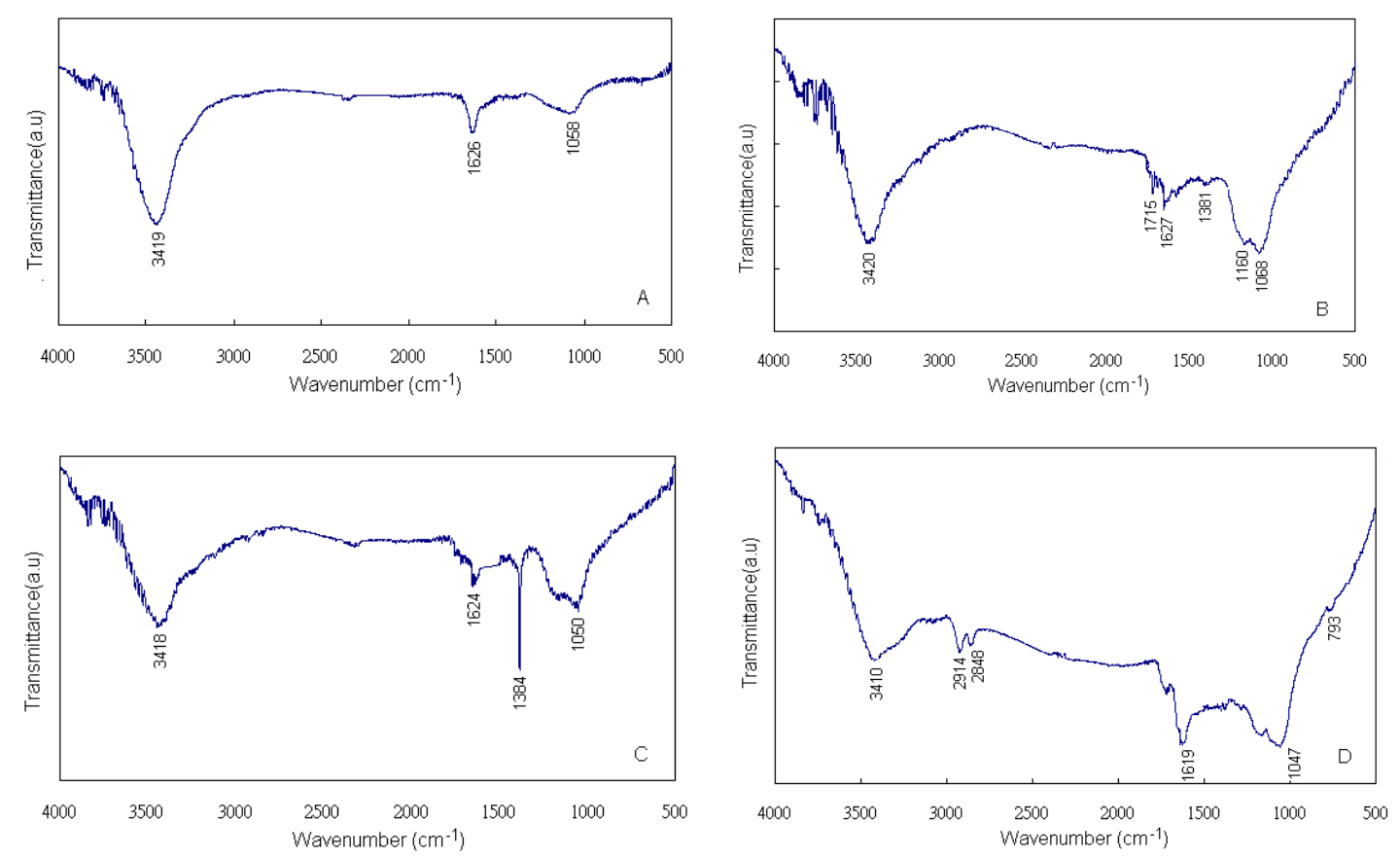

Fig. 1. FTIR spectra of the MWCNTs (A: Pristine, B: UV/O 3 -treated, C: Reduced, D: Silanized) 
In order to compare the suspension stability of the MWCNTs obtained at different processing steps, ethanol solutions with a MWCNTs concentration of $0.5 \mathrm{mg} / \mathrm{ml}$ were prepared. Figure 2 shows the results of the suspending states of the MWCNTs samples after 5 minutes of dispersion via sonication. Comparison of these photographs indicates that the suspension stability of the pristine MWCNTs (Fig. 2A) was poor: they easily sedimented in ethanol due to the agglomeration and poor hydrogen-bonding ability. The pretreated MWCNTs showed better stability than the pristine ones only in the short term (Fig. 2B, left panel), and phase separation occurred after 24 h (Fig. 2B, right panel). For the chemically modified MWCNTs (Fig. 2C-E), the functional groups on the surface of MWCNTs enhanced their hydrogen-bonding abilities. This led to the improved suspension stability compared with the pristine and pretreated ones, through the conversion of the MWCNT surface from a hydrophobic to a hydrophilic state. The MWCNTs functionalized by GPTMS (Fig. 2E, right panel) presented the best stability both in the short and long terms, due to the functionalization effect on their surface.

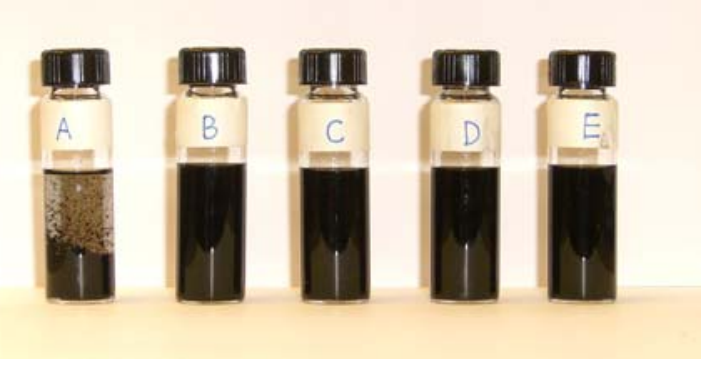

5 minutes after ultrasonication

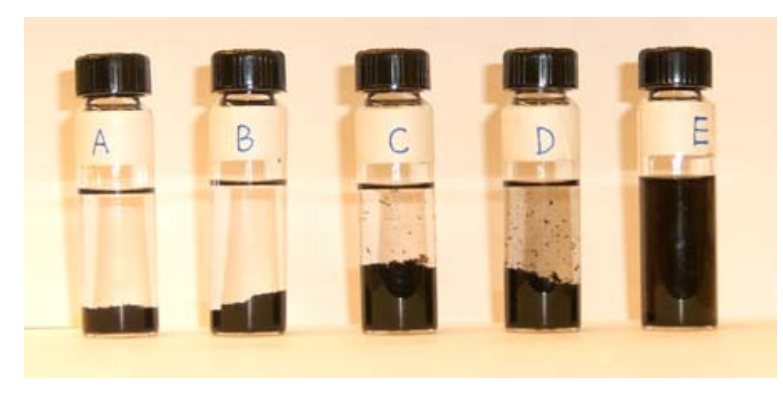

$24 \mathrm{~h}$ after ultrasonication

Fig. 2. Suspension stability of the MWCNTs (A: Pristine, B: Pretreated, C: UV/O $\mathrm{O}_{3}$-treated, D:

Reduced, E: Silanized)

Figure 3 shows the typical TEM images of the MWCNTs at different processing steps. The pristine 
MWCNTs were severely agglomerated (Fig. 3A). After the pretreatment using ball milling and ultrasonic irradiation (Fig. 3B), the agglomeration was significantly reduced. The chemically modified MWCNTs (Fig. 3C-E) showed much improved dispersion with few agglomerates. The individual MWCNTs were detached loosely without significant changes in their lengths. These results are in agreement with the observations described above in reference to Fig. 2 that the chemically modified MWCNTs exhibited improved suspension stability in ethanol.
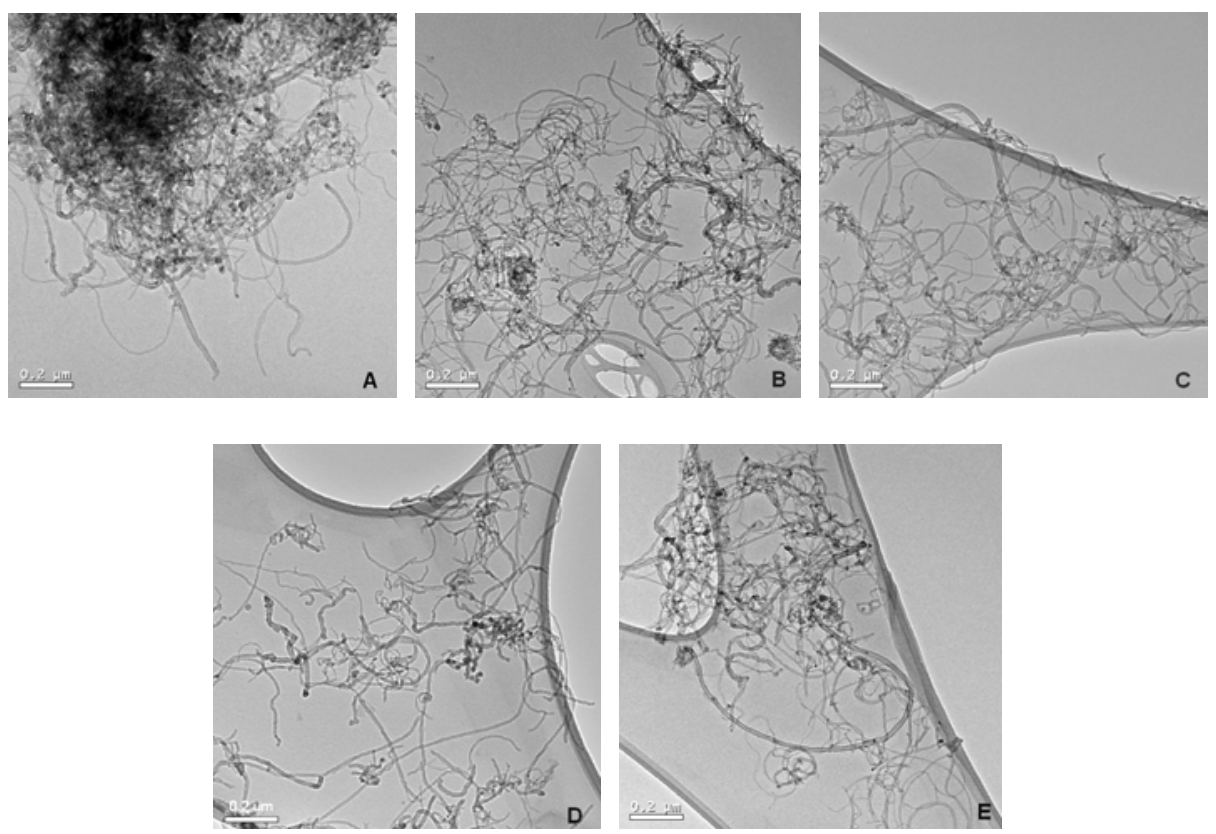

Fig. 3. Dispersion of MWCNTs (A: Pristine, B: Pretreated, C: UV/O ${ }_{3}$-treated, D: Reduced, E: Silanized).

The surface morphologies of the MWCNTs at different processing steps are shown in Figure 4. As can be seen from Fig. 4A, the end tips of the pristine MWCNT were closed. After the pretreatment through ball milling and ultrasonication (Fig. 4B), however, the end tips of many MWCNTs were open, a reflection of broken $\mathrm{C}-\mathrm{C}$ bonds along the graphene layers of the co-axial tubes. This observation is in agreement with the previous report [17]. 

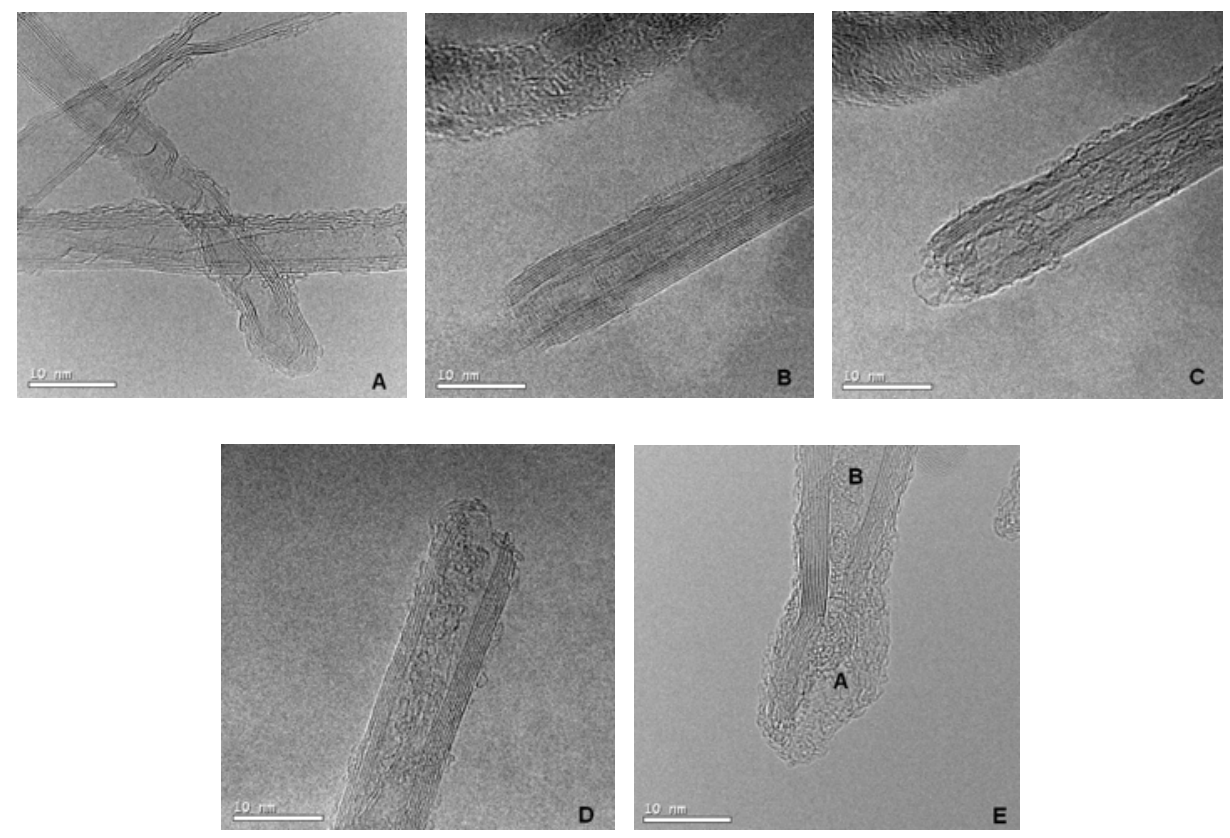

Fig. 4. Surface morphology of MWCNTs (A: Pristine, B: Pretreated, C: UV/O3-treated, D:

Reduced, E: Silanized).

The sound, layered structure of the pretreated and chemically modified MWCNTs (Fig. 4B-D) indicates that there was little damage to the MWCNTs surface. Some amorphous materials were attached to the end tips of the silane-treated MWCNTs (spot A in Fig. 4E). The detection of silicon by the EDX (Fig. 5A) confirms that these amorphous materials were derived from the silane molecules. The fact that no silicon was detected by the EDX (Fig. 5B) in the inner part of the MWCNTs (spot B in Fig. 4E) verifies that only the surface layer of the MWCNTs has been activated by our method. No GPTMS molecules have penetrated into the tubes during the silanization process. 

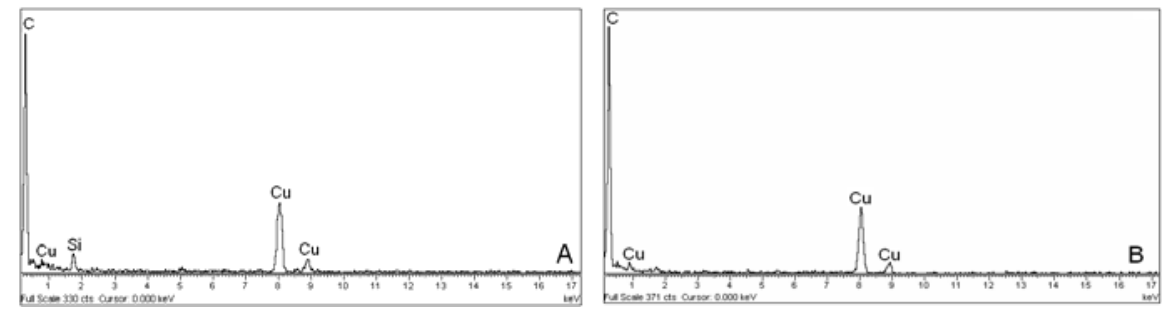

Fig. 5. EDX Spectrum of the silane-treated MWCNTs

We also investigate the possibility of the damage to the MWCNT surface which was introduced by the silanization reactions. Figure 6 shows the surface morphology of the silane-treated MWCNTs after it was kept at $400^{\circ} \mathrm{C}$ in nitrogen gas for $1 \mathrm{~h}$. This micrograph suggests that the silane molecules on the MWCNT surface can be removed (spots A and B in Fig. 6). The layered structure of the MWCNTs remained largely intact, and there was no obvious damage to the MWCNTs during the silanization process.

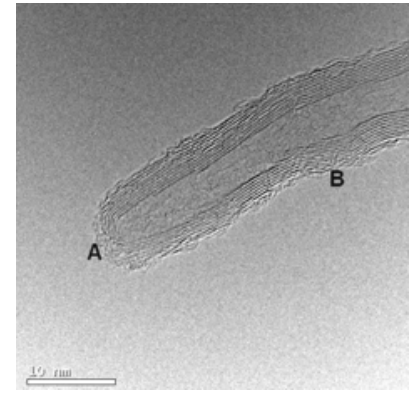

Fig. 6. Surface morphology of the MWCNTs after decomposition of silane molecules.

3.3 XPS Results 

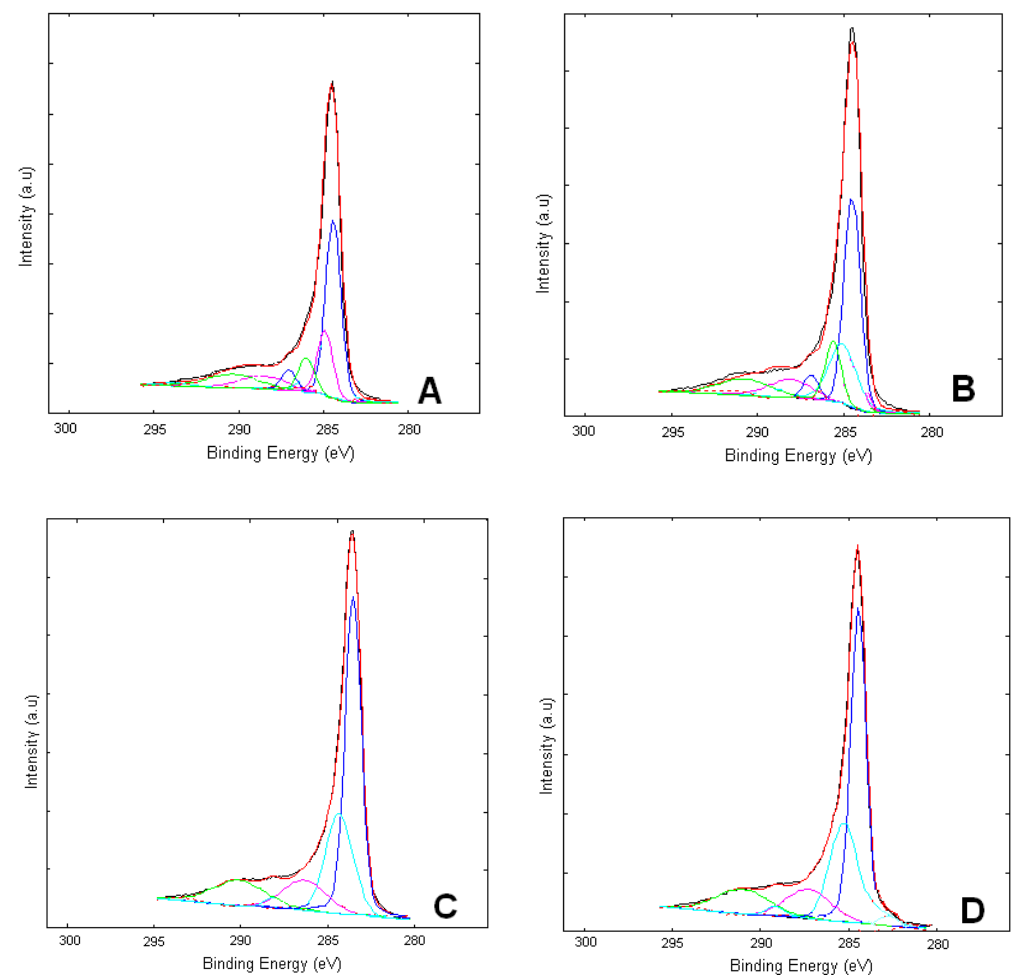

Fig. 7. C1s XPS spectrum of MWCNTs (A: Pristine, B: UV/O 3 -treated, C: Reduced, D: Silanized)

Figure 7 presents C1s XPS spectra of the MWCNTs. The C 1s peak of the pristine MWCNTs (Fig. 7A) can be deconvoluted into five fitting curves $[13,18]$ with peaks at $284.5,285.2,286.1,287.4$ and $289.4 \mathrm{eV}$. The last one at $290.7 \mathrm{eV}$ corresponds to a shakeup feature of the aromatic structure present in the MWCNTs [*]. The respective percentages and elemental compositions of the MWCNTs are summarized in Table 1 (excluding the peak at $290.7 \mathrm{eV}$ ) and Table 2.

After the $\mathrm{UV} / \mathrm{O}_{3}$ treatment (Fig. 7B), the components at $\sim 286.2$ and $\sim 289.2 \mathrm{eV}$ are attributed to the CO and COO groups, representing $9.09 \%$ and $8.67 \%$ of the carbon signal (Table 1), which are much higher than those of the pristine MWCNTs. The atomic percentage of the oxygen in the sample also increased significantly from $1.35 \%$ (pristine) to $8.74 \%$ (Table 2), indicating that the $\mathrm{UV} / \mathrm{O}_{3}$ process effectively generated more functional groups on the MWCNT surface.

* http://srdata.nist.gov/xps/index.htm, NIST standard reference database 20, accessed in March 2006 12 
The MWCNTs after reduction by $\mathrm{LiAlH}_{4}$ exhibited a different spectrum with the $\mathrm{C} 1 \mathrm{~s}$ peak (Fig. 7C). The increase in the relative intensity at $\sim 286.2 \mathrm{eV}$ along with the virtual disappearance of the peaks at $\sim 287.4 \mathrm{eV}(\mathrm{C}=\mathrm{O})$ and $\sim 289.2 \mathrm{eV}(\mathrm{COO})$ suggest the conversion of these groups into the alcohol groups (Table 1). This conversion process can be useful in anchoring the GPTMS molecules with the MWCNTs through the reaction between the hydroxyl groups and the methoxyl groups of silane. A small increase of $\mathrm{Al}$ arose from the LiAlH4 that was not completely eliminated by the washing steps.

The GPTMS-functionalized MWCNTs (Fig. 7D) showed a much similar spectrum to that of the reduced ones. However, a new contribution attributed to the Si-C appeared at $282.8 \mathrm{eV}$ along with an element composition of silicon standing at $1.68 \%$. These observations further confirm the presence of silane on the MWCNTs after the reactions.

Table 1 Summary of the relative percentage of the carbon and assignation

\begin{tabular}{ccccccc}
\hline Binding Energy $(\mathrm{eV})$ & 282.8 & 284.5 & $285.1-285.3$ & $286.0-286.5$ & $287.6-287.8$ & $289.0-289.4$ \\
and Assignation & $\mathrm{Si}-\mathrm{C}$ & $\mathrm{C}_{\mathrm{g}} \mathrm{sp}^{2}$ & $\mathrm{C}_{\mathrm{d}} \mathrm{sp}^{3}$ & $\mathrm{C}-\mathrm{O}$ & $-\mathrm{C}=\mathrm{O}$ & CO-O \\
\hline Pristine & $/$ & 73.41 & 13.28 & 5.42 & 4.01 & 3.88 \\
UV/O ${ }_{3}$-treated & $/$ & 64.35 & 13.91 & 9.09 & 3.98 & 8.67 \\
Reduced & $/$ & 62.37 & 20.52 & 12.70 & 2.56 & 1.85 \\
Silanized & 3.97 & 60.83 & 23.88 & 12.85 & 0.77 & 1.67 \\
\hline
\end{tabular}

Table 2 Summary of the element composition of the MWCNTs

\begin{tabular}{ccccccc}
\hline Element (Atom \%) & $\mathrm{C}$ & $\mathrm{O}$ & $\mathrm{Al}$ & $\mathrm{S}$ & $\mathrm{Fe}$ & $\mathrm{Si}$ \\
\hline Pristine & 98.34 & 1.35 & 0.21 & 0.04 & 0.06 & $/$ \\
$\mathrm{UV} / \mathrm{O}_{3}$-treated & 90.93 & 8.74 & 0.26 & $/$ & 0.07 & $/$ \\
Reduced & 90.64 & 8.90 & 0.40 & $/$ & 0.06 & $/$
\end{tabular}




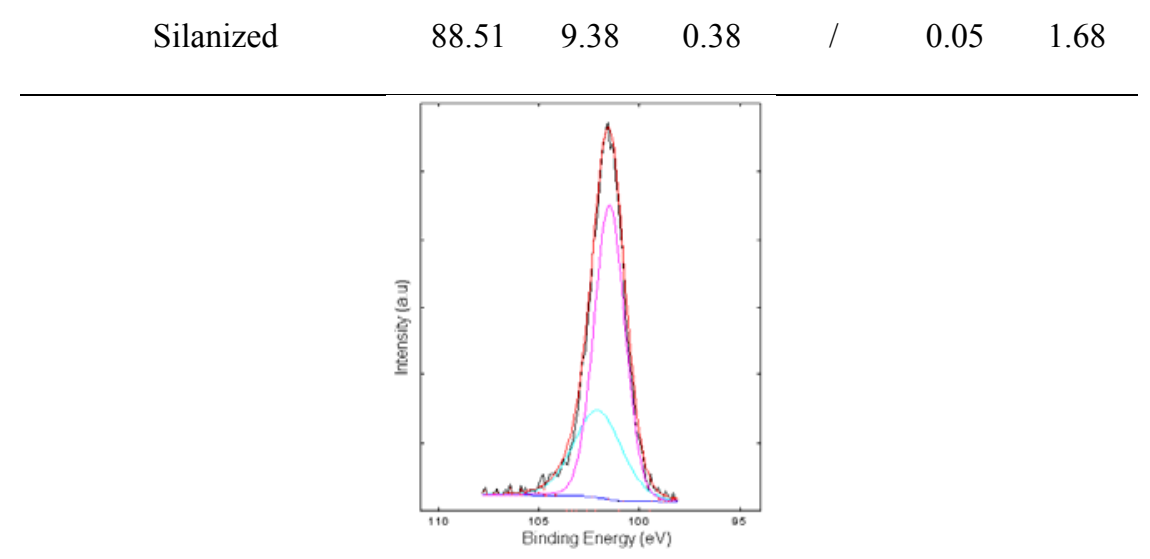

Fig. 8. Si 2p XPS spectrum of silane-treated MWCNTs

Figure 8 shows deconvoluted Si $2 p$ signal of the silane-treated MWCNTs. The two peaks at different binding energies indicate that there were two chemical states of Si. These can be explained by the reaction illustrated in Figure 9: the first one at a binding energy of $101.6 \mathrm{eV}$ represents the bond of silicon with oxygen originating from the MWCNTs (Si-O-CNT, reaction A in Fig. 9) [12]; the second one at $103.3 \mathrm{eV}$ is attributed to the siloxane $(-\mathrm{Si}-\mathrm{O}-\mathrm{Si}-)$ resulting from the partial hydrolysis of the GPTMS molecules (reaction B in Fig. 9) during the silanization reaction [12]. These results are in good agreement with the reaction mechanisms of silane, including hydrolysis of $-\mathrm{OCH}_{3}$, condensation to oligomers, hydrogen bond between oligomer and $\mathrm{OH}$ groups on the substrate, and the formation of the covalent linkage between silane and the substrate [11]. The relatively high percentage of the first contribution $(80.03 \%$ vs $19.97 \%$ ) confirms that the main reaction occurred between the GPTMS molecule and the surface of the reduced MWCNTs.

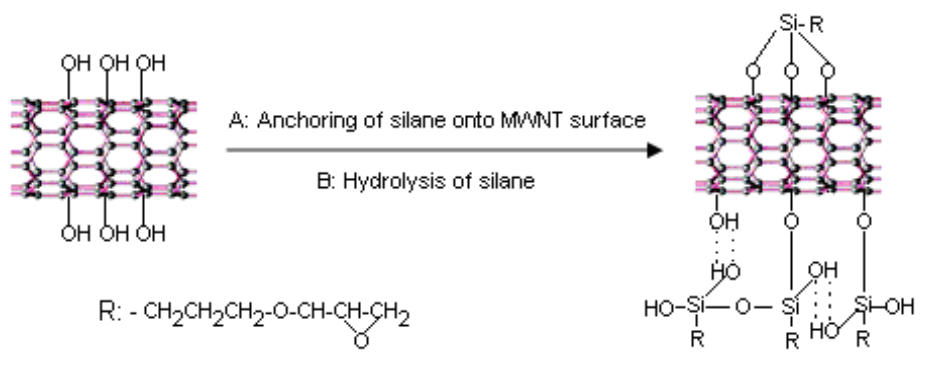


Fig. 9. Schematics of the reactions taking place between the reduced MWCNTs and the silane 4. Summary

In this work, we developed a method for the chemical functionalization of the MWCNTs through a combined process of $\mathrm{UV} / \mathrm{O}_{3}$ treatment and silanization reaction. The hydrophobic surface of the MWCNTs was converted to hydrophilic through $\mathrm{UV} / \mathrm{O}_{3}$ and silane treatments. The results indicate good dispersion of MWCNTs in ethanol and attachment of silane molecules containing epoxy end-groups on the MWCNT surface via the reaction of the - $\mathrm{OH}$ groups of the MWCNTs and the $-\mathrm{OCH}_{3}$ groups of the silane molecules. This in turn would contribute to enhancing the MWCNT's compatibility with polymer matrix, which will be the subject of forthcoming publications.

\section{Acknowledgements}

This project is supported by the Research Grant Council of Hong Kong SAR (Project number 614505). Technical assistance from the Materials Characterization and Preparation Facilities (MCPF) of HKUST is also appreciated.

\section{References}

[1]. Thostenson ET, Ren ZF, Chou TW. Advances in the science and technology of carbon nanotube and their composites: a review. Compos Sci Technol 2001; 61: 1899-912 [2]. Coleman JN, Khan U, Gun'ko YK. Mechanical reinforcement of polymers using carbon nanotubes. Adv Mater 2006; 18: 689-706

[3]. Tang BZ, Xu HY. Preparation, alignment, and optical properties of soluble poly(phenylacetylene) wrapped carbon nanotubes. Macromolecules 1999; 32: 2569-76 [4]. Fiedler B, Gojny FH, Wichmann MHG, Nolte MCM, Schulte K. Fundamental aspects of nano-reinforced composites, Compos Sci Technol (In press) 
[5]. Tasis D, Tagmatarchis N, Bianco A, Prato M, Chemistry of Carbon Nanotubes. Chem Rev 2006; 106: 1105-36

[6]. Shen K, Xu H, Jiang Y, Pietraß T. The role of carbon nanotube structure in purification and hydrogen adsorption. Carbon 2004; 42:2315-22

[7]. Monthioux M, Smith BW, Burteaux B, Claye A, Fischer JE, Luzzi DE. Sensitivity of single-wall carbon nanotubes to chemical processing: an electron microscopy investigation. Carbon 2001; 39: 1251-72

[8]. Lu X, Zhang L, Xu X, Wang N, Zhang Q. Can the sidewalls of single-wall carbon nanotubes be ozonized? J Phys Chem B 2002; 106: 2136-39

[9]. Mawhinney DB, Naumenko V, Kuznetsova A, Yates JT, Liu J, Smalley RE. Infrared spectral evidence for the etching of carbon nanotubes: ozone oxidation at 298 K. J Am Chem Soc 2000; $122: 2383-84$

[10]. Sham ML, Kim JK. Surface functionalities of multi-wall carbon nanotubes after UV/Ozone and TETA treatments. Carbon 2006; 44: 768-77

[11]. Plueddemann EP. Silane coupling agent. New York: Plenum. 1991: 115-151

[12]. Velasco-Santos C, Martınez-Hernandez AL, Lozada-Cassou M, Alvarez-Castillo A, Castano VM. Chemical functionalization of carbon nanotubes through an organosilane. Nanotechnology 2002; 13: 495-8

[13]. Vast L, Philippin G, Destree A, Moreau N, Fonseca A, Nagy JB,et al. Chemical functionalization by a fluorinated trichlorosilane of multi-walled carbon nanotubes. Nanotechnology 2004; 15: 781-5

[14]. Deng YH, Deng CH, Yang D, Wang, Fu SK, Zhang XM. Preparation, characterization and application of magnetic silica nanoparticle functionalized multi-walled carbon nanotubes. Chem Commun 2005; 44: 5548-50 
[15]. Ramanathan T, Fisher FT, Ruoff RS, Brinson LC. Amino-functionalized carbon nanotubes for binding to polymers and biological systems. Chem Mater 2005; 17: 1290-95

[16]. Sun JM. Application principle and theory of epoxy resins. Beijing: China machine press. 2002: $18-22$

[17]. Pierard N, Fonseca A, Konya Z, Willems I, Tendeloo GV, Nagy JB. Production of short carbon nanotubes with open tips by ball milling. Chem Phys Lett 2001; 335: 1-8 [18]. Ago H, Kugler T, Cacialli F, Salaneck WR, Shaffer MSP, Windle AH, Friend RH. Work functions and surface functional groups of multiwall carbon nanotubes. J Phys Chem B 1999; 103: 8116-21. 


\section{List of Captions for figures and Tables.}

Figure 1 FTIR spectra of the MWCNTs (A: Pristine, B: UV/Oㄱ-treated, C: Reduced, D: Silanized)

Figure 2 Suspension stability of the MWCNTs (A: Pristine, B: Pretreated, C: UV/O근 -treated, D:

Reduced, E: Silanized)

Figure 3 Dispersion of MWCNTs (A: Pristine, B: Pretreated, C: UV/O 3 -treated, D: Reduced, E:

Silanized).

Figure 4 Surface morphology of MWCNTs (A: Pristine, B: Pretreated, C: UV/O3-treated, D:

Reduced, E: Silanized).

Figure 5 EDX Spectrum of the silane-treated MWCNTs

Figure 6 Surface morphology of the MWCNTs after decomposition of silane molecules.

Figure $7 \mathrm{C} 1 \mathrm{~s}$ XPS spectrum of MWCNTs (A: Pristine, B: UV/O ${ }_{3}$-treated, C: Reduced, D:

Silanized)

Figure 8 Si 2p XPS spectrum of silane-treated MWCNTs

Figure 9 Schematics of the reactions taking place between the reduced MWCNTs and the silane

Table 1 Summary of the relative percentage of the carbon and assignation

Table 2 Summary of the element composition of the MWCNTs 